\title{
Formation and Disruption of Globular Star Clusters
}

\author{
S. Michael Fall
}

Space Telescope Science Institute, 3700 San Martin Drive, Baltimore, $M D$ 21218, USA

\section{Qing Zhang}

Space Telescope Science Institute, 3700 San Martin Drive, Baltimore, $M D$ 21218, USA

\begin{abstract}
.
In the first part of this article, we review observations of the mass and luminosity functions of young and old star cluster systems. We also review some of the physical processes that may determine the characteristic mass of globular clusters and the form of their mass function. In the second part of this article, we summarize our models for the disruption of clusters and the corresponding evolution of the mass function. Much of our focus here is on understanding why the mass function of globular clusters has no more than a weak dependence on radius within their host galaxies.
\end{abstract}

\section{Background}

The most basic attribute of any population of astronomical objects is its mass function. In our notation, $\psi(M)$ is the number of objects per unit mass $M$, and $\Psi(\log M)$ is the number of objects per unit $\log M$. These two forms of the mass function are related by $\Psi(\log M)=(\log e)^{-1} M \psi(M)$. They contains information about the physical processes involved in the formation and subsequent evolution of the objects. There are well-known reasons, for example, why stars and galaxies have roughly the masses they do and not some others, even if we lack definitive theories for the detailed forms of the stellar and galactic mass functions. This article addresses the question: What physical processes determine the mass function of star clusters, especially that of globular clusters?

The upper panel of Figure 1 shows the empirical mass function of young star clusters in the interacting and merging Antennae galaxies (from Zhang \& Fall 1999). This function declines monotonically, approximately as $\psi(M) \propto M^{-2}$, over the entire observed range, $10^{4} \lesssim M \lesssim 10^{6} M_{\odot}$. In a young cluster system, such as the one in the Antennae galaxies, where the spread in the ages of the clusters is comparable with their median age, the luminosity function need not reflect the underlying mass function, since the clusters have a wide range of mass-to-light ratios. However, luminosity functions are easier to determine than mass functions and are known for more cluster systems. For all the young cluster systems studied so far, the luminosity functions are well-approximated by power laws (Milky Way, van den Bergh \& Lafontaine 1984; LMC, Elson \& Fall 1985; 


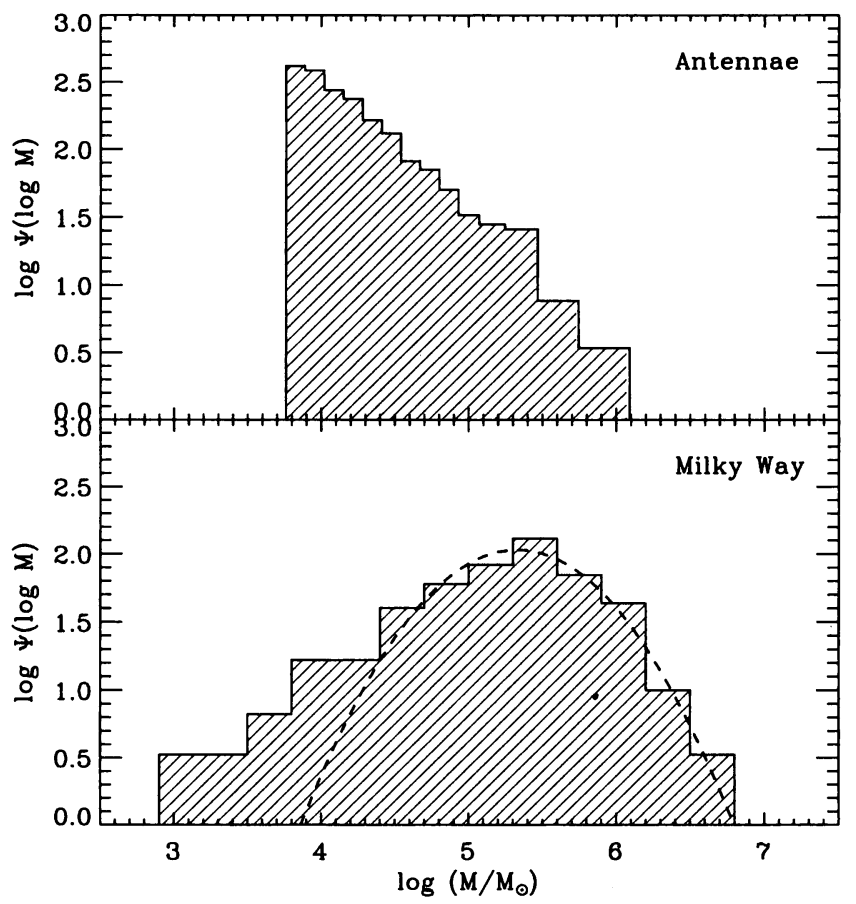

Figure 1. Empirical mass functions of young star clusters in the Antennae galaxies and old globular clusters in the Milky Way. The former is from Zhang \& Fall (1999); the latter is based on data compiled by Harris $(1996,1999)$. The dashed curve is the usual lognormal representation of the mass function, corresponding to a Gaussian distribution of magnitudes.

M33, Christian \& Schommer 1988; Antennae, Whitmore et al. 1999). In fact, the mass and luminosity functions of young star clusters are remarkably similar to the mass function of interstellar clouds in the Milky Way (Dickey \& Garwood 1989; Solomon \& Rivolo 1989), as emphasized by several authors (Harris \& Pudritz 1994; Elmegreen \& Efremov 1997).

The lower panel of Figure 1 shows the empirical mass function old globular clusters in the Milky Way. This was derived from the luminosities of all the clusters in the Harris $(1996,1999)$ compilation, with a fixed mass-to-light ratio $\left(M / L_{V}=3\right)$, since the spread in the ages of the clusters is smaller than their median age. The mass function of the globular clusters in the Milky Way, like those in the spheroids of other well-studied galaxies, rises to a peak or turnover at $M_{p} \approx 2 \times 10^{5} M_{\odot}$ and then declines. The corresponding feature in the luminosity function, at $\bar{M}_{V} \approx-7.3$, is sometimes used as a standard candle in distance determinations. The empirical mass function is often represented by a lognormal function, although, as Figure 1 indicates, the former is actually shallower than the latter for small masses. The crucial point here is that the mass and luminosity functions of young cluster systems are scale-free, whereas those of old cluster systems have a preferred scale. Why is this? 


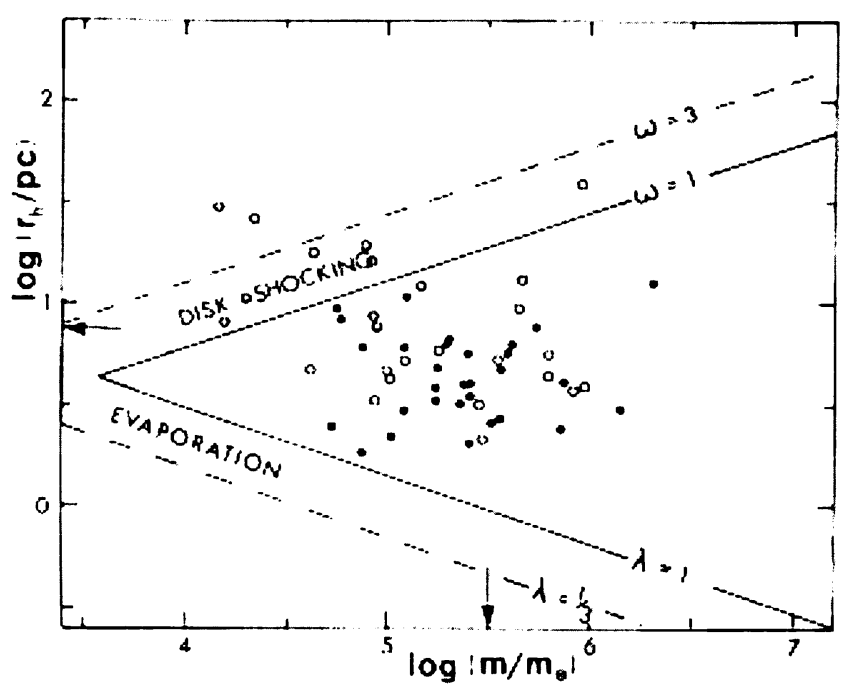

Figure 2. Survival triangle in the mass-radius plane from Fall \& Rees (1977). The solid lines show where the timescales for disruption by disk shocks and two-body relaxation are equal to the Hubble time. The dashed lines indicate some of the uncertainties in these timescales. The dots represent globular clusters in the Milky Way; filled and open symbols indicate clusters closer to and farther from the Galactic center than the Sun (about $8 \mathrm{kpc}$ ). Clusters outside the triangle will be destroyed within the next Hubble time, whereas those inside will survive for longer.

Two explanations have been proposed for the age-dependence of the mass functions. The first is that the conditions in ancient galaxies and protogalaxies favored the formation of clusters with masses $\sim 10^{5}-10^{6} M_{\odot}$ but that these conditions no longer prevail in modern galaxies. For example, the Jeans mass could have been much higher in the past, as a result of less efficient cooling by heavy elements and/or more efficient dissociation of molecular hydrogen (Fall \& Rees 1985; Kang et al. 1990). It is sometimes stated in observational papers that this theory is ruled out by the lack of correlation between the luminosities (masses) of globular clusters and their metallicities. However, this argument is not correct, because the dependence of the Jeans mass on the abundances of heavy elements and molecules is essentially bimodal. The relevant Jeans mass is determined by whether or not gas in the protoclusters cools rapidly at temperatures below $10^{4} \mathrm{~K}$. If it does, the Jeans mass is $\lesssim 10^{2} M_{\odot}$; if it does not, the Jeans mass is $\sim 10^{6} M_{\odot}$. In the first case, one is making stellar associations or small open clusters; in the second, one is making globular clusters.

The other explanation for the differences between the mass functions of young and old cluster systems is that they represent the erosion of the initial mass function by the gradual disruption of low-mass clusters. Star clusters are relatively weakly bound objects and are vulnerable to disruption by a variety of processes, including mass loss by stellar evolution (supernovae, stellar winds, 
etc.), evaporation by two-body relaxation and gravitational shocks (against the galactic disk and bulge), and dynamical friction (see Spitzer 1987 for a review). Figure 2 shows the survival region for globular clusters in the mass-radius plane defined by some of these processes (from Fall \& Rees 1977). Recent work shows that disruptive processes, especially two-body relaxation, operating for a Hubble time, would cause the mass function to evolve from a variety of initial forms into one resembling that of old globular clusters (Vesperini 1997, 1998; Baumgardt 1998; Fall \& Zhang 2001).

There is, however, a potentially serious objection to the idea that disruption is responsible for the low-mass form of the mass function of old globular clusters: the chief disruptive processes operate at different rates in different parts and different types of galaxies (Caputo \& Castellani 1984; Gnedin \& Ostriker 1997). For example, the rate at which stars escape by two-body relaxation depends on the density of a cluster, which is determined by the tidal field, and hence is higher in the inner parts of galaxies than in the outer parts. The rate at which stars escape by gravitational shocks is also higher in the inner parts of galaxies because the orbital periods are shorter and the surface density of the disk is higher there. Moreover, disks are absent in elliptical galaxies. Thus, if the mass function were strongly affected by disruptive processes, one might expect its form to depend on radius within a galaxy and to vary from one galaxy to another. This, however, is contradicted by many observations showing that the mass function of old globular clusters varies little, if at all, within and among galaxies (Harris 1991).

\section{New Models}

With these issues in mind, we have developed some new models to compute the evolution of the mass function of star cluster systems (Fall \& Zhang 2001). Our models include disruption by two-body relaxation, gravitational shocks, and stellar evolution. We describe these processes by approximate formulae that can be solved largely analytically. The clusters are assumed to orbit in a galactic potential that is static, spherical, and has a logarithmic dependence on the distance $R$ from the galactic center. Each cluster is assume to be tidally limited at the pericenter of its orbit and to lose mass at a constant mean internal density. The population of orbits is specified by the distribution function $f(E, J)$, defined as the number of clusters per unit volume of position-velocity space with energy and angular momentum per unit mass near $E$ and $J$. The distribution function determines how much the clusters are mixed in radius and hence how much the mass function varies with radius.

We consider two simple models for the initial distribution function. The first is the Eddington model

$$
f_{0}(E, J) \propto \exp \left(-E / \sigma^{2}\right) \exp \left[-1 / 2\left(J / R_{A} \sigma\right)^{2}\right] .
$$

This has velocity dispersions $\sigma_{R}=\sigma$ and $\sigma_{T}=\sigma\left[1+\left(R / R_{A}\right)^{2}\right]^{-1 / 2}$ in the radial and transverse directions, where the anisotropy radius $R_{A}$ marks the transition from a nearly isotropic to a predominantly radial velocity distribution. The second initial distribution function we consider has the form

$$
f_{0}(E, J) \propto \exp \left(-E / \sigma^{2}\right) J^{-2 \beta} .
$$




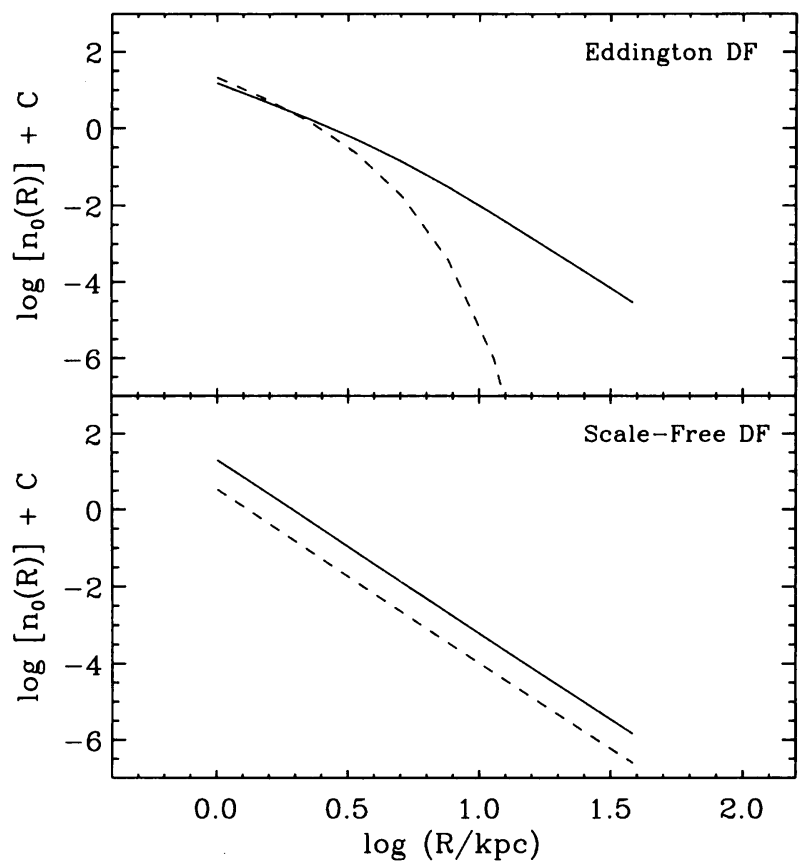

Figure 3. Initial densities of clusters positions (solid lines) and pericenters (dashed lines) for the Eddington and scale-free distribution functions. Note that the distribution of pericenters is narrower than the distribution of cluster positions for the Eddington model but not for the scale-free model.

In this case, the radial and transverse velocity dispersions are $\sigma_{R}=\sigma$ and $\sigma_{T}=\sigma(1-\beta)^{1 / 2}$. We refer to this as the scale-free model. In most cases, we adopt $R_{A}=5 \mathrm{kpc}$ and $\beta=0.5$, so that both models have the same velocity anisotropy at the median radius of the globular cluster system $\left(R_{h}=5 \mathrm{kpc}\right)$. For our purposes, the most important difference between the Eddington and scalefree models is that, in the former, the velocity anisotropy increases outward, whereas in the latter, it is the same at all radii. Thus, the distribution of pericenters is narrower in the Eddington model than it is in the scale-free model, as shown in Figure 3.

We consider four models for the initial mass function of the clusters: (1) a pure power law, $\psi_{0}(M) \propto M^{-2},(2)$ the same power law truncated at $M_{l}=3 \times$ $10^{5} M_{\odot},(3)$ a Schechter function, and (4) a lognormal function. Figure 4 shows the evolution of the mass function, averaged over all radii, for the Eddington initial distribution function. In all four cases, the mass function develops a peak, which, after $12 \mathrm{Gyr}$ is remarkably close to the observed peak, despite the very different initial conditions. Below the peak, the evolution is dominated by two-body relaxation, and the mass function always develops a low-mass tail of the form $\psi(M)=$ const. This can be traced to the fact that, in the late stages of disruption, the masses of tidally limited clusters decrease linearly with time. The predicted low-mass form of the mass function agrees nicely with the 


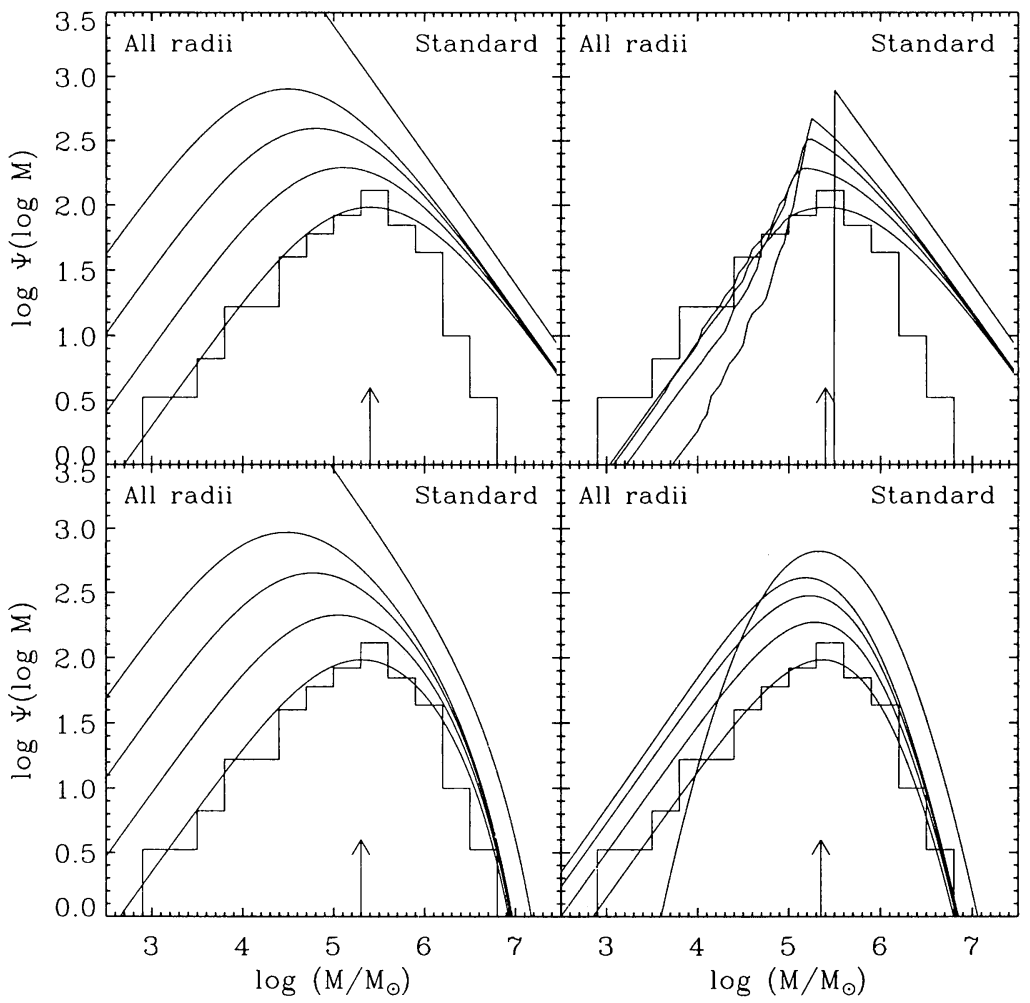

Figure 4. Evolution of the mass function, averaged over all radii, for the Eddington initial distribution function and four different initial mass functions. These are (clockwise from upper left): a pure power law, a truncated power law, a Schechter function, and a lognormal function. Each mass function is plotted at $t=0,1.5,3,6$, and $12 \mathrm{Gyr}$; the arrows indicate the peak at $t=12 \mathrm{Gyr}$. The histograms depict the empirical mass function of globular clusters in the Milky Way (the same as in Fig. 1). Note that the peak mass in the models is similar to that in the observations for the four different initial conditions. 


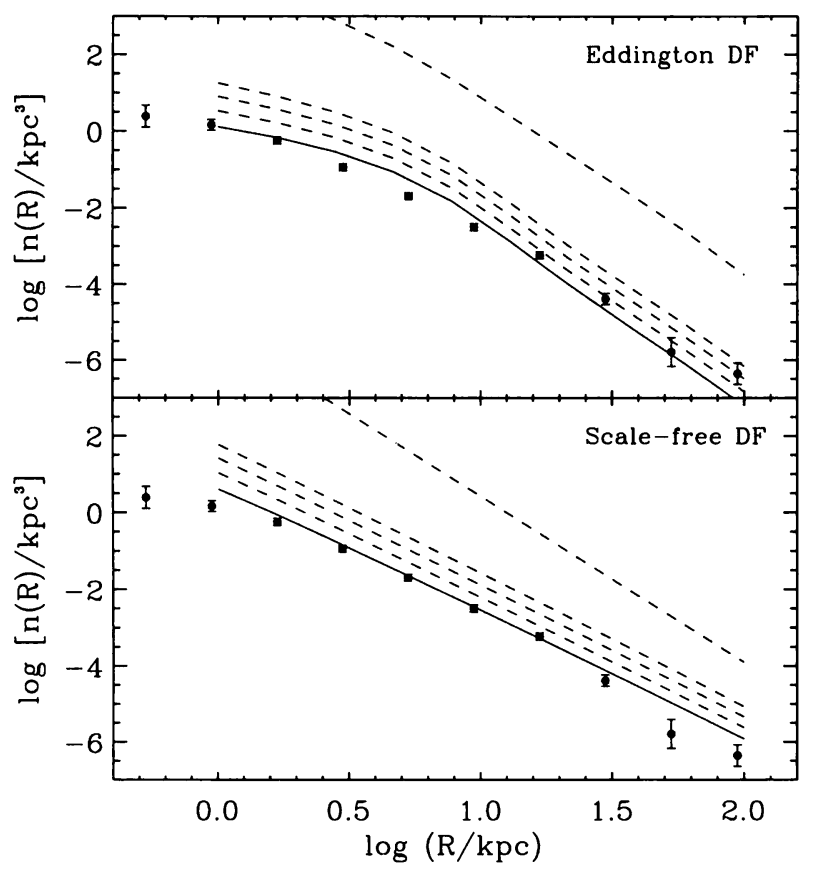

Figure 5. Evolution of the number density profile of the cluster system for the Eddington and scale-free initial distribution functions. The profiles are plotted at $t=0,1.5,3,6$, and 12 Gyr. The data points depict the empirical profile for globular clusters in the Milky Way. Note that the final profiles in the models are in reasonable agreement with the empirical profile.

observed form. Above the peak, the evolution of the mass function is dominated by stellar evolution at early times and by gravitational shocks at late times. These processes shift the mass function to lower masses but leave its shape nearly invariant. Thus, the present shape of the mass function at high masses is largely determined by its initial shape. The radially averaged mass function for the scale-free model (not shown) is similar to that for the Eddington model. The evolution of the density profiles of the cluster systems for both models are shown in Figure 5.

Where the Eddington and scale-free models differ most is in the radial variation of the mass function of the clusters. This is shown in Figures 6 and 7, which display the evolution separately for clusters inside and outside $R=5 \mathrm{kpc}$. In both models, the peak mass is larger at small radii. This is caused mainly by the higher rate of evaporation by two-body relaxation, resulting from the larger mean densities of clusters with small pericenter distances. In the Eddington model, the radial variation of the peak mass is weak enough to be consistent with observations, whereas in the scale-free model, the variation is too strong. The reason for this is that the distribution of pericenters is narrower in the 


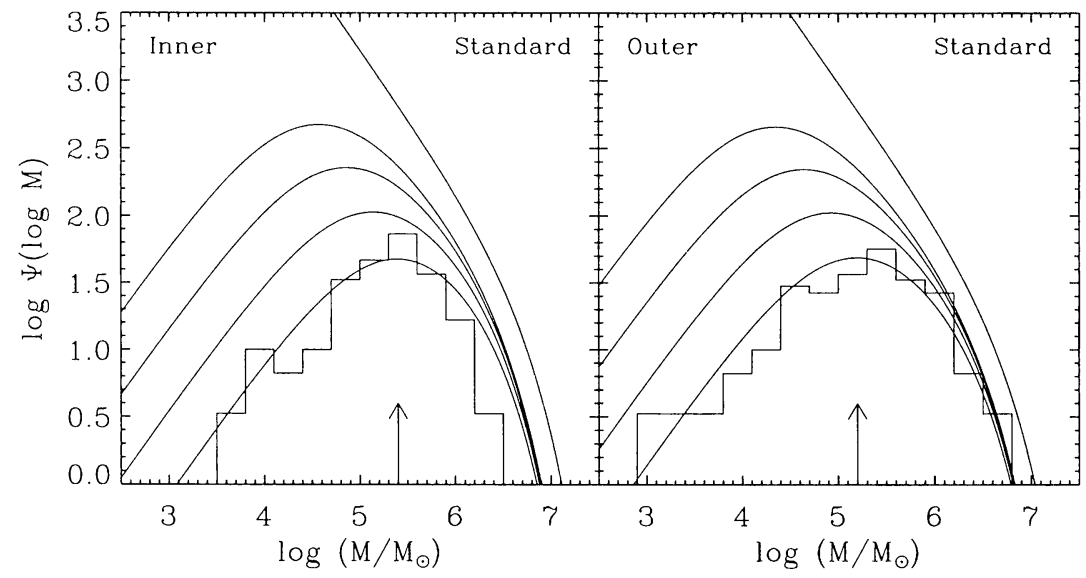

Figure 6. Evolution of the mass function, averaged over inner radii $(R<5 \mathrm{kpc})$ and outer radii $(R>5 \mathrm{kpc})$, for the Eddington initial distribution function and the Schechter initial mass function. Each mass function is plotted at $t=0,1.5,3,6$, and $12 \mathrm{Gyr}$; the arrows indicate the peak at $t=12$ Gyr. The histograms depict the empirical mass functions of globular clusters in the Milky Way in the corresponding ranges of radii. Note that the shift in the peak mass in the models between inner and outer radii is relatively small.

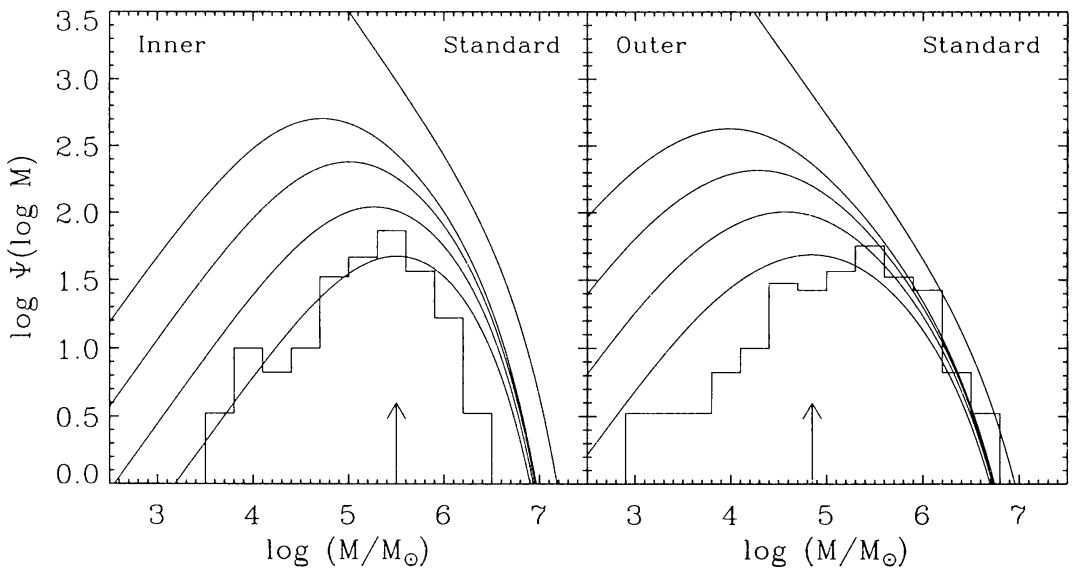

Figure 7. Evolution of the mass function, averaged over inner radii $(R<5 \mathrm{kpc})$ and outer radii $(R>5 \mathrm{kpc})$, for the scale-free initial distribution and the Schechter initial mass function. Each mass function is plotted at $t=0,1.5,3,6$, and $12 \mathrm{Gyr}$; the arrows indicate the peak at $t=12$ Gyr. The histograms depict the empirical mass functions of globular clusters in the Milky Way in the corresponding ranges of radii. Note that the shift in the peak mass in the models between inner and outer radii is relatively large. 
Eddington model, leading to a smaller range of disruption rates, than in the scale-free model.

The lesson here is that radial mixing is a necessary but not sufficient condition for weak radial variations in the mass function of globular clusters. The other requirement is that the radial anisotropy in the initial velocity distribution of the clusters increase outward, as in the Eddington model. The present velocity distribution of globular clusters in the Milky Way appears to have little or no radial anisotropy (Frenk \& White 1980; Dinescu, Girard, \& van Altena 1999). This is qualitatively what we would expect, since most clusters on elongated orbits would already have been destroyed, leaving behind a nearly isotropic or tangentially biased velocity distribution. These conclusions are based on models with static, spherical galactic potentials, in which each cluster returns to the same pericenter on each of its revolutions around a galaxy. In galaxies with time-dependent and/or non-spherical potentials, however, the pericenters of the clusters may change from one revolution to the next. This will tend to homogenize the disruption rates of the clusters and hence to weaken the radial variation in their mass function. Whether these effects are significant in galaxies like the Milky Way is not yet known.

It is important to test the models of disruption against observations. A clear prediction of the models is that the peak mass $M_{p}$ should increase with the ages of clusters. This might be observable in galaxies in which clusters formed continuously over long periods of time. Alternatively, the evolution of the peak mass might be observable in galaxies with bursts of cluster formation at different times, such as in a sequence of merger remnants. This test may be difficult, however, because the luminosity corresponding to the peak mass is relatively small for young clusters (since $M_{p}$ varies more rapidly with $t$ than $M / L_{V}$ does). Another prediction of the models is that the peak mass should decrease with increasing distance from the centers of galaxies, unless this has been completely diluted by the mixing of pericenters mentioned above. Searches for radial variations in the peak mass have so far been inconclusive. This test is difficult because the diffuse light of the host galaxies also varies with radius, making it harder to find faint clusters in the inner regions. Finally, the strong dependence of the peak mass on the ages of clusters and the weak dependence on their positions within and among galaxies cast some doubt on the use of the peak luminosity as a standard candle for distance estimates. This method may be viable, however, if the samples of clusters are carefully chosen from similar locations in similar galaxies.

The models of disruption help to dissolve some of the boundaries in the classification of star clusters. The shape of the mass function above the peak is largely preserved as clusters are disrupted and hence should reflect processes at the time they formed. Below the peak, however, the shape of the mass function is determined entirely by disruption, mainly driven by two-body relaxation, and hence contains no information about how the clusters formed. If there were any feature in the initial mass function, such as a Jeans-type lower cutoff, it would have been erased. In our models, the only feature in the present mass function, the peak at $2 \times 10^{5} M_{\odot}$, is largely determined by the condition that clusters of this mass have a timescale for disruption comparable to the Hubble time. Thus, it is conceivable that star clusters of different types (open, populous, 
globular, etc.) formed by the same physical processes with the same initial mass function and that the differences in their present mass functions reflect only their different ages and local environments, primarily the strength of the galactic tidal field. Our results therefore support the suggestion that at least some of the star clusters formed in merging and other starburst galaxies may be regarded as young globular clusters. Further investigations of these objects may shed light on the processes by which old globular clusters formed.

\section{References}

Baumgardt, H. 1998, A\&A, 330, 480

Caputo, F., \& Castellani, V. 1984, MNRAS, 207, 185

Christian, C. A., \& Schommer, R. A. 1988, AJ, 95, 704

Dickey, J. M., \& Garwood, R. W. 1989, ApJ, 341, 201

Dinescu, D. I., Girard, T. M., \& van Altena, W. F. 1999, AJ, 117, 1792

Elmegreen, B. G., \& Efremov, Y. N. 1997, ApJ, 480, 235

Elson, R. A. W., \& Fall, S. M. 1985, PASP, 97, 692

Fall, S. M., \& Rees, M. J. 1977, MNRAS, 181, 37P

Fall, S. M., \& Rees, M. J. 1985, ApJ, 298, 18

Fall, S. M., \& Zhang, Q. 2001, ApJ, in press (astro-ph/0107298)

Frenk, C. S., \& White, S. D. M. 1980, MNRAS, 193, 295

Gnedin, O. Y., \& Ostriker, J. P. 1997, ApJ, 474, 223

Harris, W. E. 1991, ARA\&A, 29, 543

Harris, W. E. 1996, AJ, 112, 1487

Harris, W. E. 1999, http://www.physics.mcmaster.ca/Globular.html

Harris, W. E., \& Pudritz, R. E. 1994, ApJ, 429, 177

Kang, H., Shapiro, P. R., Fall, S. M., \& Rees, M. J. 1990, ApJ, 363, 488

Solomon, P. M., \& Rivolo, A. R. 1989, ApJ, 339, 919

Spitzer, L. 1987, Dynamical Evolution of Globular Clusters (Princeton: Princeton Univ. Press)

van den Bergh, S., \& Lafontaine, A. 1984, AJ, 89, 1822

Vesperini, E. 1997, MNRAS, 287, 915

Vesperini, E. 1998, MNRAS, 299, 1019

Whitmore, B. C., Zhang, Q., Leitherer, C., Fall, S. M., Schweizer, F., \& Miller, B. W. 1999, AJ, 118, 1551

Zhang, Q., \& Fall, S. M. 1999, ApJ, 527, L81

\section{Discussion}

J. Holtzman: Can you comment on the applicability of your models to elliptical galaxies? 
M. Fall: The models should apply to cluster systems in elliptical galaxies when the disk shocking is shut off. How to apply the models to galaxies with different masses or circular velocities is not yet entirely clear. It depends, among other things, on the so-called baryonic Tully-Fisher and Faber-Jackson relations, which are still somewhat controversial.

C. Grillmair: First, isn't disk shocking pretty severe for globular clusters? Second, tidal stripping is fairly inefficient (per orbit), so the progression of the peak in the globular cluster luminosity function towards the present value may be even slower than your calculations indicate.

M. Fall: First, two-body relaxation is the dominant disruptive process for lowmass clusters (relative to the peak in the mass function), while gravitational shocks and stellar evolution are the dominant processes for high-mass clusters. Second, the effect you mention may in fact occur, but it would only delay the evolution by a few orbital periods, which, for most clusters, amounts to a small fraction of the Hubble time.

E. Vesperini: In order to avoid a radial gradient in the mean mass not consistent with observations, you need to have enough radial mixing; on the other hand, the cluster concentrations, core and half-mass radii, slope of the stellar mass function are correlated with the galactocentric distance, and at least some of these trends are likely to be due to the effects of dynamical evolution. How do you reconcile the existence of these trends with radial mixing?

M. Fall: Interesting question. If the radial mixing were complete, it would wash out all the correlations you mention. But the radial mixing need not be complete to bring the radial variation in the mass function within observational tolerances. In fact, the distribution of pericenters in the Eddington model, while narrower than that in the scale-free model, is still wide enough to permit strong correlations between many properties of the clusters and their galactocentric radii. 